\title{
3D Printing Hydrogel-Based Soft and Biohybrid Actuators: A Mini-Review on Fabrication Techniques, Applications, and Challenges
}

\author{
Wenhuan Sun ${ }^{1}$, Saul Schaffer ${ }^{1}$, Kevin Dai ${ }^{1}$, Lining Yao ${ }^{2}$, Adam Feinberg $^{3}$ and \\ Victoria Webster-Wood ${ }^{1 *}$
}

${ }^{1}$ Biohybrid and Organic Robotics Group, Department of Mechancial Engineering, Carnegie Mellon University, Pittsburgh, PA, United States, ${ }^{2}$ Morphing Matter Lab, Human-Computer Interaction Institute, School of Computer Science, Carnegie Mellon University, Pittsburgh, PA, United States, ${ }^{3}$ Regenerative Biomaterials and Therapeutics Group, Department of Materials Science and Engineering, Carnegie Mellon University, Pittsburgh, PA, United States

\section{OPEN ACCESS}

Edited by:

Stefano Palagi,

Sant'Anna School of Advanced

Studies, Italy

Reviewed by:

Mahmut Selman Sakar,

École Polytechnique Fédérale de

Lausanne, Switzerland

Ivan Rehor,

University of Chemistry and Technology in Prague, Czechia

${ }^{*}$ Correspondence:

Victoria Webster-Wood vwebster@andrew.cmu.edu

Specialty section: This article was submitted to Soft Robotics,

a section of the journal Frontiers in Robotics and Al

Received: 27 February 2021 Accepted: 14 April 2021

Published: 29 April 2021

Citation:

Sun W, Schaffer S, Dai K, Yao L, Feinberg $A$ and Webster-Wood $V$ (2021) 3D Printing Hydrogel-Based Soft and Biohybrid Actuators: A MiniReview on Fabrication Techniques,

Applications, and Challenges.

Front. Robot. Al 8:673533.

doi: 10.3389/frobt.2021.673533
Stimuli-responsive hydrogels are candidate building blocks for soft robotic applications due to many of their unique properties, including tunable mechanical properties and biocompatibility. Over the past decade, there has been significant progress in developing soft and biohybrid actuators using naturally occurring and synthetic hydrogels to address the increasing demands for machines capable of interacting with fragile biological systems. Recent advancements in three-dimensional (3D) printing technology, either as a standalone manufacturing process or integrated with traditional fabrication techniques, have enabled the development of hydrogel-based actuators with on-demand geometry and actuation modalities. This mini-review surveys existing research efforts to inspire the development of novel fabrication techniques using hydrogel building blocks and identify potential future directions. In this article, existing 3D fabrication techniques for hydrogel actuators are first examined. Next, existing actuation mechanisms, including pneumatic, hydraulic, ionic, dehydration-rehydration, and cell-powered actuation, are reviewed with their benefits and limitations discussed. Subsequently, the applications of hydrogel-based actuators, including compliant handling of fragile items, micro-swimmers, wearable devices, and origami structures, are described. Finally, challenges in fabricating functional actuators using existing techniques are discussed.

Keywords: 3D printing, hydrogel, biohybrid actuator, fabrication, actuation mechanism

\section{INTRODUCTION}

Recent advances in 3D printing have enabled the production of customizable hydrogel-based actuators with a variety of applications. Hydrogels are hydrophilic and porous crosslinked polymer networks whose mechanical, chemical, and stimulation-responsive properties can be tuned based on composition and manufacturing processes (Shi et al., 2019). Such properties enable applications of hydrogels in several domains, including tissue engineering (Billiet et al., 2012), drug delivery (Li and Mooney, 2016), wound dressings (Varaprasad et al., 2020), and soft robotics (Lee et al., 2020; Wallin et al., 2018). Recent reviews are available on the use of hydrogels in biomedical applications (Banerjee et al., 2018; Champeau et al., 2020), polymeric shape memory hydrogels and hydrogel actuators (Shang et al., 2019), and biomimetic hydrogel actuators (Le et al., 2019). Readers interested in 
comprehensive reviews on hydrogel-based 3D printing for biomedical applications can refer to (Li et al., 2020). However, a focused synopsis of the fabrication techniques and challenges for $3 \mathrm{D}$ printing hydrogel-based actuators has not been previously reported.

To address this gap, this mini-review presents recent advancements in $3 \mathrm{D}$ printing for fabricating hydrogel actuators, either as a standalone manufacturing process or integrated with traditional fabrication techniques, where actuators are defined as any components that perform defined movements or geometric changes. Here, we present characteristic examples of existing 3D printing techniques for hydrogel actuator fabrication based on their roles in the fabrication process: direct $3 \mathrm{D}$ printing; mixed-mode $3 \mathrm{D}$ printing, where $3 \mathrm{D}$ printed hydrogel structures undergo additional post-printing steps; and the use of $3 \mathrm{D}$ printing to fabricate intermediate tools. Additionally, we discuss the strengths and limitations of $3 \mathrm{D}$ printed hydrogel actuators based on their actuation modalities. Applications of 3D printed hydrogel actuators are subsequently surveyed. Through this discussion, we provide a focused survey on the challenges and limitations in fabricating hydrogel actuators using existing $3 \mathrm{D}$ printing techniques to inspire future hydrogel fabrication advances that improve the performance of hydrogel-based actuators.

\section{3D PRINTING TECHNIQUES FOR HYDROGEL ACTUATOR FABRICATION}

3D printing enables hydrogel actuator fabrication either through direct printing of actuator structures, mixed-mode printing of hydrogels with other functional materials, or through printing intermediate tools. Several direct 3D printing technologies have emerged including inkjet-based, laser-based, and extrusion-based approaches. Other fabrication techniques can be added to direct printing to create mixed-mode fabrication platforms, such as combining $3 \mathrm{D}$ printing and hydrogel electrospinning. Additionally, 3D printing can be used to rapid prototype molds for casting hydrogels. Each approach has challenges and advantages that should be considered when selecting fabrication techniques.

\subsection{Direct 3D Printing of Hydrogel Actuators 2.1.1 Inkjet-Based 3D Printing}

Inkjet-based hydrogel 3D printing uses computer-controlled layer-by-layer deposition of droplets onto a substrate (Figure 1A1) (Axpe and Oyen, 2016). A variety of shape changing hydrogel actuators that respond to external stimuli have been inkjet printed (Peng and Wang, 2018). For example, utilizing the swelling response of inkjet printed UV-curable polyacrylated monomers that undergo layer-by-layer UVpolymerization, water-swellable joints can be fabricated to enable modular 4D self-reconfiguration following printing (Raviv et al,. 2014). Alternatively, inkjet printers can be used to introduce reactive inks into hydrogel systems to induce locally active regions. For example, metal ion ink to induce anisotropic crosslinking density and controlled deformation during swelling
(Figure 1A2) (Peng et al., 2017), and reactive catalyst ink made of bis(2,2'-bipyridine)-4'-methyl-4-carboxybipyridine-ruthenium $\mathrm{N}$-succinimidyl ester-bis(hexafluorophosphate) (Ru (sbpy)) to create user-defined reactive regions that experience oxidation state-dependent swelling ratio (Kramb et al., 2014).

Hydrogels with low viscosity of $<10 \mathrm{mPa}$.s (Axpe and Oyen, 2016) are typically used in inkjet printing and the resulting hydrogel actuators have low structural integrity (Zolfagharian et al., 2017a). The aforementioned techniques produced relatively thin and simple hydrogel structures. Fabricating thick hydrogel actuators using inkjet printing remains a challenge. To overcome this limitation, reinforcing material, such as collagen sheets or agarose rods, can be printed as support materials before depositing hydrogel layers (Jakab et al., 2010). Recent advancement in freeform liquid support-based inkjet printing techniques may further lift the geometric constraints on $3 \mathrm{D}$ inkjet printed hydrogel actuators. For example, (Christensen et al., 2015) fabricated vascular-like structures with overhanging features by directly inkjet printing hydrogel into a calcium chloride solution, which serves as both a crosslinker and support.

\subsubsection{Laser-Based 3D Printing}

Hydrogel actuators with 2D geometry can be fabricated with classic photolithography that uses photomasks for geometry control, such as stop-flow lithography (Rehor et al., 2020). Hydrogel structures with complex 3D geometry can be made via laser-based $3 \mathrm{D}$ printing techniques from three major categories: laser-induced forward transfer, which uses laser energy to discharge hydrogel droplets from a donor layer onto a substrate (Guillemot et al., 2011); two photon-polymerization (2PP) (Figure 1B1) which initiates hydrogel polymerization through irradiation with near-infrared laser pulses (Billiet et al., 2012); and stereolithography (SLA) (Figure 1C1), which selectively crosslink photo-sensitive monomer resin using scanning UV laser beams (Cvetkovic et al., 2014). A variation of SLA is digital light processing (DLP), which projects the entire image of a layer on the resin using UV (or visible) light from a digital projector (Soman et al., 2013). High resolution SLA provides a variety of tunable fabrication parameters and can be used to fabricate hydrogel actuators with controllable motion through asymmetry. Specifically, anisotropic swelling behavior has been encoded by asymmetric high surface area patterning, asymmetric crosslinking density, and asymmetric chemical composition by tuning SLA parameters (Odent et al., 2019), (Figures 1C2,3). Compared with other hydrogel 3D printing techniques, 2PP shows exceptional spatial resolution and is advantageous for building miniature actuators and robots. For example, (Xiong et al., 2011) fabricated ion-responsive hydrogel microcantilever actuators with swift motion (millisecond response time) and small size (10 $\mu \mathrm{m}$ cantilever length) using 2PP (Figure 1B2).

Laser-based hydrogel 3D printing techniques show great spatial resolution (up to submicron resolution (Ovsianikov et al., 2010; Xing et al., 2015). However, they are typically subject to high costs due to expensive hardware and software and the limitation of printing small features. 

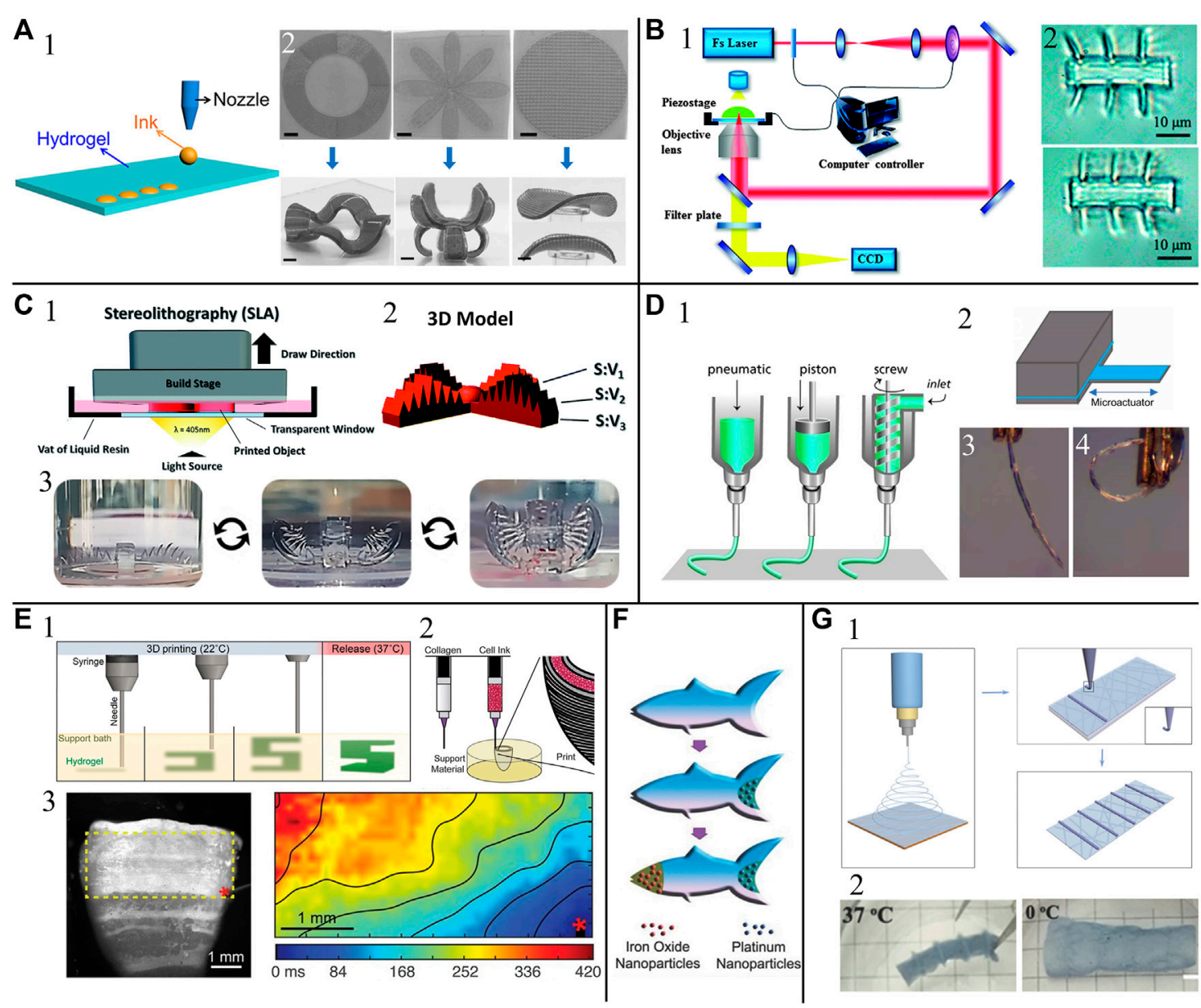

FIGURE 1 | Examples of hydrogel actuators fabricated with (A) inkjet printing, (B) two-photon polymerization printing (2PP), (C) stereolithography (SLA), (D) extrusion-based printing, (E) embedded printing, and (F,G) mixed-mode 3D printing. (A1) The inkjet printing process. (A2) Hydrogel actuators with inkjet printed patterns to achieve controlled 3D deformation. Adapted from (Peng and Wang 2018) @2018 John Wiley and Sons, Inc. (B1) A typical 2PP process induced by a nearinfrared femtosecond laser. Reprinted from (Xing et al., 2015) @2015 the Royal Society of Chemistry. (B2) 2PP printed hydrogel actuator in water (top) and in $1 \mathrm{M}$ $\mathrm{NaCl}$ solution (bottom). Reprinted from (Xiong et al., 2011) @2011 the Royal Society of Chemistry (C1) The SLA process (C2) 3D model of an SLA printed hydrogel actuator with varying surface area to volume ratio. (C3) Osmotically driven actuation of the actuator. Adapted from (Odent et al., 2019 ) (O2019 the Royal Society of Chemistry. (D1) Common extrusion-based printing process. Reprinted from (Malda et al., 2013) O2013 John Wiley and Sons, Inc. (D2) A bending actuator with 3D printed humidity-responsive hydrogel layer (blue) with water vapor induced actuation (D3-4). Adapted from (Tyagi et al., 2020) @2020 The Authors under the CC-BY 4.0 license. (E1) Embedded printing process where the thermo-responsive support bath exhibits Bingham plastic properties during printing and melt at raised temperature for printed component release. Reprinted from (Hinton et al., 2015) @2015 The Authors under the CC-BY 4.0 license (E2) Fabrication process of an embedded printed human cardiac ventricle model using collagen and cell ink. (E3) (left) Point stimulation of the model stained with calcium-sensitive dye. (right) Color-coded calcium mapping of a subregion showing anisotropic calcium wave propagation during stimulated contraction. Adapted from (Lee et al., 2019) O2019 AAAS. (F) Fabrication of a 3D printed microfish with encapsulated magnetic nanoparticles for motion control and catalytic Pt nanoparticles for self-propulsion powered by $\mathrm{H}_{2} \mathrm{O}_{2}$ decomposition. Adapted from (Zhu et al., 2015) @2015 John Wiley and Sons, Inc. (G1) A hydrogel actuator fabricated with hydrogel electrospinning and 3D printing. (G2) The actuator exhibits temperature-dependent curvature. Adapted from (Chen et al., 2018) @2018 John Wiley and Sons, Inc. All figures are used with permission.

\subsubsection{Extrusion-Based 3D Printing}

Extrusion-based 3D printing dispenses hydrogel filament through a nozzle onto a substrate using a piston, pneumatic pump or screw (Figure 1D1) (Li et al., 2020). With a large selection of open-source and low-cost fabrication hardware and software, extrusion-based 3D printing is considered attractive for hydrogel processing (Li et al., 2020), and many hydrogel actuators fabricated with extrusion printing have been reported. Using a pneumatically driven fluid dispenser, (Tyagi et al., 2020) fabricated a microactuator where an active hydrogel layer that swells upon hydration was bonded with a passive gel layer to create a cantilever structure with humidity-induced bending capability (Figures 1D2-4). With continuous hydrogel filament feeding, extrusion-based $3 \mathrm{D}$ printing provides a convenient way to create anisotropic hydrogel features that facilitate directional actuation. For example, (Gladman et al., 2016) printed composite hydrogel actuators with anisotropic swelling behavior by aligning cellulose fibrils along predefined 
printing pathways. (Cheng et al., 2019) demonstrated the versatility of extrusion-based 3D printing with modified hydrogels by fabricating fluidic and stimulus-activated actuators, including an artificial tendril with phototropic motion.

Extrusion-based 3D printing is a cost-effective hydrogel fabrication technique that is compatible with hydrogel ink with viscosities ranging from 30 to $6 \cdot 10^{7} \mathrm{mPa} \cdot \mathrm{s}$ (Kyle et al., 2017). Large hydrogel constructs can be printed with moderate spatial resolution. With recent advances in support material for embedded 3D printing, the spatial resolution of extrusion based 3D printed hydrogel actuators can be further improved (see Section 2.1.4).

\subsubsection{Embedded 3D Printing}

$3 \mathrm{D}$ printing overhanging features without supporting structures often leads to low print fidelity due to unwanted deformation. This problem is more pronounced for hydrogel actuator fabrication because many of the inks are extremely soft, e.g., alginate and collagen, and can not even support their own weight. Recently, several embedded $3 \mathrm{D}$ printing techniques have been proposed to resolve the aforementioned issue by printing modified inks into a support bath (Zhang et al., 2021; Mirdamadi et al., 2020). Notably, (Hinton et al., 2015) printed 3D structures by embedding printed hydrogels within a gelatin-based thermoreversible bi-phase support material, which provides support during printing and liquefies when the temperature is raised post-printing for part retrieval (Figure 1E1), coined Freeform Reversible Embedding of Suspended Hydrogels (FRESH). Using FRESH 2.0 with improved spatial resolution $(20 \mu \mathrm{m}$ filament resolution), (Lee et al., 2019) printed cardiac ventricles with synchronized contractions using human cardiomyocytes and collagen (Figures 1E2,3).

\subsection{Mixed-Mode 3D Printing and 3D Printing of Intermediate Tools}

Combining 3D printing with other fabrication techniques (mixed-mode), allows 3D printed hydrogel actuator fabrication with additional functionality. For example, (Zhu et al., 2015) demonstrated a chemically powered and magnetically guided microfish, where the hydrogel body was fabricated via UV photolithography. Catalytic Pt nanoparticles were then encapsulated at the tail via crosslinking $\mathrm{Pt}$-doped resin to induce self-propulsion via decomposition of $\mathrm{H}_{2} \mathrm{O}_{2} . \quad \mathrm{Fe}_{3} \mathrm{O}_{4}$ nanoparticles were loaded into the head with a similar method for magnetic control (Figure 1F). In another example of mixedmode fabrication, (Chen et al., 2018) combined hydrogel electrospinning and extrusion-based hydrogel printing to fabricate actuators with temperature-responsive bending behavior induced by the difference in properties of electrospun and 3D printed hydrogels (Figure 1G). (Cvetkovic et al., 2014) demonstrated a biobot whose hydrogel body was SLA 3D printed and actuated by a strip of engineered mammalian skeletal muscle cast over the printed hydrogel body.

In addition to direct $3 \mathrm{D}$ printing, hydrogel actuators can be made with methods that indirectly involve 3D printing. For example, (Yuk et al., 2017) fabricated hydraulic hydrogel actuators whose bodies were made by casting within $3 \mathrm{D}$ printed molds. In addition to mold fabrication for hydrogel casting, components of hydrogel actuators have been $3 \mathrm{D}$ printed using non-hydrogel materials with desired properties. For example, (Mestre et al., 2019) fabricated a biohybrid actuator using a 3D printed PDMS skeleton that serves as an actuation force indicator and structural support. Combining 3D printing with other fabrication techniques facilitates rapid prototyping of actuators (Yuk et al., 2017) and integration of different materials that enable new actuation modalities (Zhu et al., 2015). However, added fabrication complexity can incur challenges, such as the necessity to switch between resins with different additives (Zhu et al., 2015) and the need to bond cast components (Yuk et al., 2017).

\section{ACTUATION MODALITIES}

Hydrogel actuators commonly rely on heterogeneous structure and/or stimuli to generate meaningful movement (Zhang and Khademhosseini, 2017). Heterogeneous structure may be generated from rational design of hydrogel geometry or from material property gradients created by dopants within the hydrogel (Odent et al., 2019). Heterogeneous stimulation may come from external humidity or $\mathrm{pH}$ gradients or optical, thermal, or electrical stimuli (Zolfagharian et al., 2017a). Actuation of these hydrogels occurs due to thermodynamic imbalance between osmosis and hydrophobicity of hydrogel polymers which causes swelling and de-swelling (Peng and Wang, 2018). However, other actuation modalities such as cell-powered, pneumatic/hydraulic, acoustic, and magnetic actuation do not typically rely on swelling behavior. Readers interested in a further review of hydrogel-based actuation mechanisms, including actuators that have not been $3 \mathrm{D}$ printed or fabricated with $3 \mathrm{D}$ printed tooling, may also be interested in (Erol et al., 2019).

\subsection{Osmotic Actuation (pH, Humidity, Ionic, Thermal, Optical)}

Many of the actuation methods of stimuli-responsive hydrogels rely on structural swelling and de-swelling driven by osmoticpressure changes in response to external stimuli such as $\mathrm{pH}$ (Nadgorny et al., 2016), humidity, temperature, electric field, and optical lasers (Yuk et al., 2017). These actuation methods are often used in 4D printing, where hydrogel structures deform over time from their original geometry into new configurations with or without further active actuation (Adam et al., 2021; Momeni et al., 2017; Spiegel et al., 2020). To mitigate the heavy computational costs of simulating 4D printed configurations, (Huang et al., 2020) proposed a modular design of shapemorphing structures with discretized building blocks using a pH-responsive bilayer hydrogel (Jin et al., 2020). For actuating hydrogels using electric field or electrolytes, hydrogel polymers can be created with ionic dopants or precursors (Zolfagharian et al., 2017b). Alternatively, hydrogels can also be actuated without external electric field by changing their chemical environment and building multi-layer hydrogels where each layer has a different swelling response to an electrolyte or chemical solution (Zheng et al., 2018). 
Hydration and dehydration cycles can drive structural expansion and contraction of hydrogel actuators due to water absorption from external humidity or immersion in water. This actuation modality is often used to create bilayer and trilayer bending hydrogel actuators. For example, (Rivera et al., 2020) fabricated a hydrogel-textile bilayer composite by 3D printing $\kappa$-carrageenan, a nontoxic hydrogel derived from red seaweed that swells in response to water, onto textile substrates. By tuning hydrogel patterns and concentrations, smart textile actuators with programmed actuation states were achieved. (Wang et al., 2017) 3D printed a B. subtilis cell-agar hydrogel mixture that experienced volume change during hydration and dehydration by utilizing cells' hygromorphic phenomenon, where a cell's size can change by $50 \%$ when varying the relative humidity. Similarly, (Yao et al., 2015) printed natto cell-water solution onto humidityinert textile substrates to create smart wearables that open up ventilation holes in response to raised skin temperature. In addition, ( $\mathrm{Lv}$ et al., 2018) used 2PP to create hydrogels with microstructure arrays of various geometries to mimic stomata found in plants. Hygroscopic swelling and deswelling causes the stomata microstructures to close and open.

Temperature changes and temperature gradients can be used to actuate hydrogels with heterogeneous lower or upper critical solution temperature (LCST, UCST) (Hua et al., 2019) and hydrogels with temperature-dependent moisture content (Hamedi et al., 2016). (Odent et al., 2019) used SLA to 3D print multi-layer hydrogel actuators that opened and closed between $25-50^{\circ} \mathrm{C}$, as well as another hydrogel actuator that was controlled by changes in $\mathrm{pH}$. (Lin et al., 2020) 3D printed a bilayer hydrogel actuator with $\alpha$-cyclodextrin polyrotaxane that both deflected and changed opacity when subjected to temperature changes.

Optical lasers can also be used to control hydrogel actuators, commonly with a photothermal process using photoresponsive hydrogels and near-infrared light (Watanabe et al., 2002; Hippler et al., 2019). With embedded light-absorbing particles, such as graphene oxide nanoparticles (Zhao et al., 2018; Breuer et al., 2019), copper sulfide nanoparticles (Pan et al., 2021), and gold nanorods (Nishiguchi et al., 2020), the photothermal process converts light energy to heat and follows the same LCST or UCST process as thermally responsive hydrogel actuators, but may be more precisely controlled with optical stimuli (Zheng et al., 2020). The swelling properties of optically stimulated, thermoresponsive hydrogels can be tuned by varying printing density to achieve ultrafast actuation (Nishiguchi et al., 2020).

\subsection{Cell-Powered Actuation}

Hydrogels are among the most common scaffold material in tissue engineering (Mantha et al., 2019). As a consequence, with the growth of biohybrid robotics research over the past decades (Webster-Wood et al., 2017), numerous cell-powered, hydrogel actuators have been developed. Biohybrid devices rely on contraction of engineered or explanted tissue to drive actuation (Ricotti et al., 2017; Webster-Wood et al., 2017; Sun et al., 2020). Cells can be cultured as monolayers to drive bendingbased actuation of bilayer cantilevers (Marzban and Yuan, 2016; Chan et al., 2012; Ricotti and Fujie, 2017), or can be cultured as three dimensional tissues using casting (Cvetkovic et al., 2014; Raman et al., 2016) or 3D printing (Mestre et al., 2019). Although many example of mobile hydrogel devices are now available in the literature (Ricotti et al., 2017; Webster-Wood et al., 2017; Sun et al., 2020; Morimoto and Takeuchi, 2020), challenges remain both in identification of optimal hydrogel composition and stiffness, and in scaling up cell-powered actuators for macroscale applications (Won et al., 2020). Despite these challenges, cell-powered actuation of hydrogels posses many unique advantages through the inclusion of material that is selfhealing, renewable, and adaptable.

\subsection{Pneumatic/Hydraulic Actuation}

Pneumatic and hydraulic actuation is based on pressure gradient across an actuator membrane induced by a gas or fluid. Compared to hydrogel actuators with osmotic swelling/ de-swelling-based actuation and cell-powered actuation, hydraulically driven hydrogel actuators are less common but show higher actuation force and/or speed (Yuk et al., 2017). These actuators have direct applications in robotics. For example, (Cheng et al., 2019) fabricated hydraulically driven tentacles using alginate-doped acrylamide hydrogel precursors. More recently, (Mishra et al., 2020) demonstrated SLA 3D-printed hydrogel bending actuators that can be pneumatically and hydraulically actuated while maintaining a stable actuator temperature via autonomic perspiration to achieve a trade-off between actuation efficiency and cooling capacity.

\subsection{Magnetic Actuation}

Hydrogels with embedded ferromagnetic nanoparticles can be actuated remotely using an external magnetic field without need for direct contact (Podstawczyk et al., 2020). (Chin et al., 2017) used a combination of photolithography and casting to demonstrate magnetically actuated hydrogel drug delivery devices and gated valves with iron oxide nanoparticles. Additionally, magnetic particle-embedded hydrogels can be 2PP printed into miniature helices with swimming capabilities under rotational magnetic fields (Ceylan et al., 2019; Bozuyuk et al., 2018; de Marco et al., 2019; Park et al., 2019; Cabanach et al., 2020) fabricated similar structures by casting magnetic nanoparticles-gelatin mixture in $2 \mathrm{PP}$ printed molds. Alternatively, such microrobots can be made by surface coating of $2 \mathrm{PP}$ printed hydrogel helix with magnetic nanoparticles (Wang et al., 2018; Koepele et al., 2020; Dong et al., 2020).

\subsection{Acoustic Actuation}

Similar to magnetic actuation, acoustic energy can be utilized to remotely manipulate micro/nano objects in fluid with high precision (Matouš et al., 2019; Guo et al., 2016; Ozcelik et al., 2018; Ren et al., 2019; Tao et al., 2019; Fornell et al., 2019). Hydrogel structures that are acoustically stimulated at their resonant frequency can manipulate fluids for mixing (Orbay et al., 2018) or generating fluid flow (Kaynak et al., 2020). In addition, (Son et al., 2020) constructed a remotely controlled hybrid gripper with both magnetic and acoustic actuation, where 

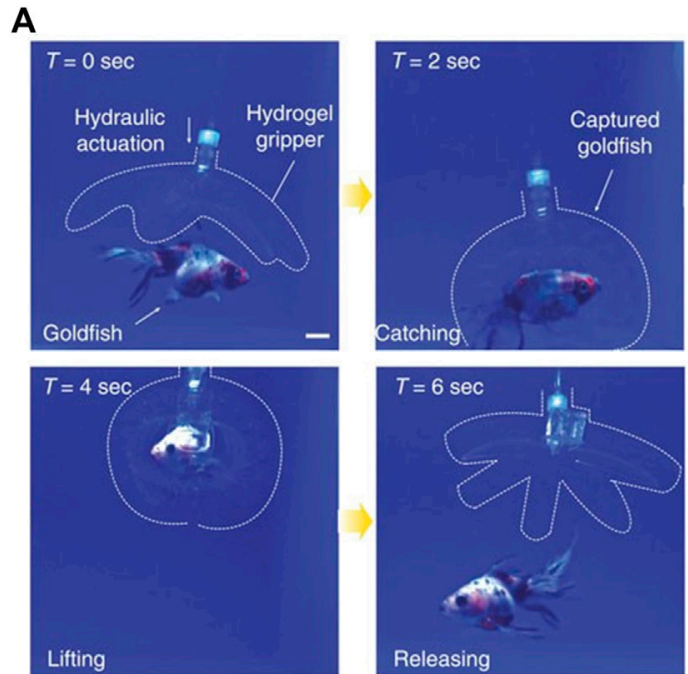

C
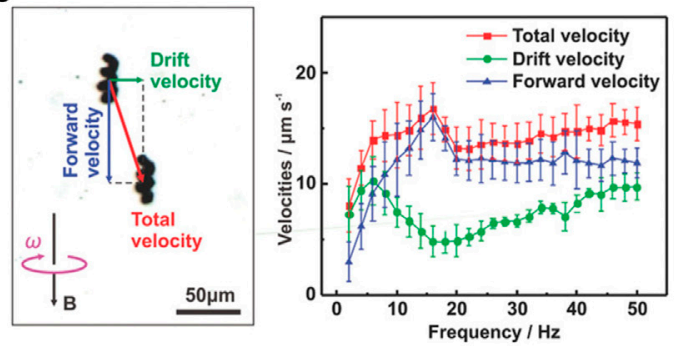

B
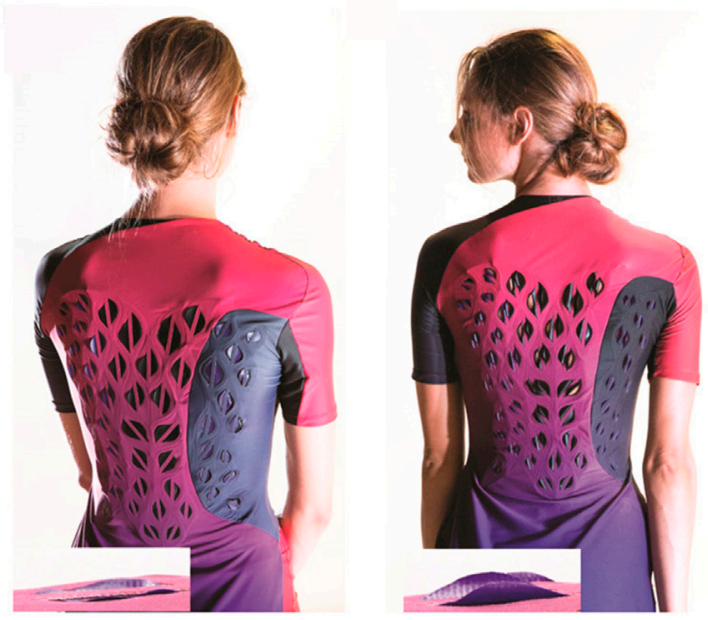

D

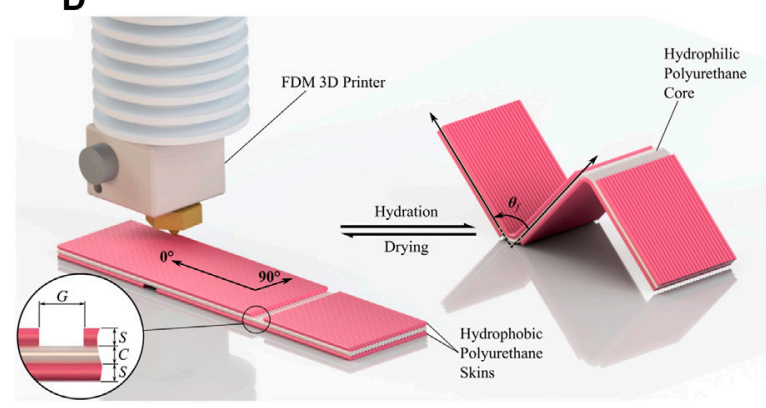

FIGURE 2 | Examples of various applications of hydrogel actuators whose fabrication involves 3D printing. (A) A hydrogel gripper catches, lifts and releases a live goldfish without harm. Dotted lines indicates the boundaries of transparent hydrogel structure. Adapted from (Yuk et al., 2017 ) @2017 The Authors under the CC-BY 4.0 license. (B) Garment prototype with sweat activated cooling ventilation showing flat ventilation flaps before exercise (left) and curved ventilation flaps after exercise (right). Adapted from (Wang et al., 2017) (02017 The Authors under the CC-BY 4.0 license. (C) A hydrogel helical micro swimmer actuated by magnetic field (left). Swimming velocities of the microswimmers at different rotational frequency at an applied magnetic rotating field of $8 \mathrm{mT}$. Adapted from (Wang et al., 2018 ) C2018 John Wiley and Sons, Inc. (D) 3D printed tri-layer actuator as modularized origami structure consist of hydrophobic polyurethane top and bottom skins (pink), with a hydrophilic polyurethane core (white) (left). It shows hydration-dependent bending at skin gaps (right). Adapted from (Baker et al., 2019 ) (O2019 The Authors under the CC-BY 4.0 license. All figures are used with permission.

the acoustically responsive hydrogel converted ultrasound energy to heat for temperature-sensitive swelling/de-swelling.

\section{SOFT ROBOTIC APPLICATIONS OF 3D PRINTED HYDROGEL ACTUATORS}

The actuation modalities described above have been used for myriad applications (Li et al., 2020). The most relevant to the field of soft robotics are manipulation and locomotion. Additionally, these actuators have use cases in wearables and as origami structures.

\subsection{Manipulators and Locomotors}

Devising a gripper to demonstrate the functionality of a fabrication technique has become a mainstay in the field of soft robotics, and the subfield of $3 \mathrm{D}$ printed hydrogel actuators is no exception. The main mode of operation for these finger-like grippers is the reversible bending of compliant beams to grasp and release objects. For example, (Mishra et al., 2020) made use of a variably porous hydrogel layer that enabled sweating of their finger actuator when introduced to hot environments to facilitate thermoregulation, which is vital for both biological and engineered systems to function at peak power for prolonged durations. A morphologically similar gripper was described by (Yuk et al., 2017) and was able to grasp and move a live fish using its fingers while remaining nearly transparent (Figure 2A), made possible by the low stiffness and visibility of the hydrogels that comprised the gripper.

3D printed hydrogel actuators have also been used to power locomotion in soft robots. Similar to grippers, these locomotory actuators operate by the cyclic deformation of beams, as demonstrated by (Han et al., 2018) for walking and (Tognato et al., 2019) for swimming. In the case of cell-powered systems, the $3 \mathrm{D}$ printed hydrogel serves as a compliant structure that is 
bent by living materials to achieve locomotion, as demonstrated by (Pagan-Diaz et al., 2018; Mestre et al., 2019; Cvetkovic et al., 2014). Beyond walking locomotion, (Wang et al., 2018) demonstrated a micro-swimming robot using $3 \mathrm{D}$ printed hydrogel actuators (Figure 2C).

\subsection{Wearables and Origami Devices}

Skin-interfacing materials require low stiffness, making $3 \mathrm{D}$ printed hydrogel actuators well-suited as wearable devices. These devices provide users with functional and esthetic utility, as shown by (Wang et al., 2017) where the sweat of the wearer triggers the opening of cooling vents on clothing (Figure 2B). Similarly, (Rivera et al., 2020) demonstrated a hydrogel-textile bilayer actuator that contracts when dehydrated, with applications as weathertriggered signage.

Origami structures couple the ease of planar fabrication methods with the geometric complexity achievable through folding. Printed as flat structures, origami-actuated hydrogels can assume complex 3D forms, as demonstrated by (Naficy et al., 2017; Gladman et al., 2016; Baker et al., 2019), from boxes to helices. These compliant, biocompatible, biodegradable, foldable structures morph between distinct configurations in response to external stimulus (Figure 2D).

\section{DISCUSSION}

Many 3D printing techniques are capable of creating hydrogel structures with user-defined geometry and responsiveness to environmental cues. Using combinations of $3 \mathrm{D}$ printing methods and hydrogels, functioning actuators with a variety of actuation modalities and potential applications have been fabricated. This mini-review surveyed the applications of $3 \mathrm{D}$ printing for hydrogel actuator fabrication and discussed their corresponding strengths and limitations. However, the applications of these actuators in soft robotics beyond the lab remain limited due, in part, to the mechanical properties of hydrogels and the available spatial

\section{REFERENCES}

Adam, G., Benouhiba, A., Rabenorosoa, K., Clévy, C., and Cappelleri, D. J. (2021). 4D Printing: Enabling Technology for Microrobotics Applications. Adv. Intell. Syst. 2000216, 2000216. doi:10.1002/aisy.202000216

Axpe, E., and Oyen, M. (2016). Applications of Alginate-Based Bioinks in 3D Bioprinting. Ijms 17, 1976. doi:10.3390/ijms17121976

Baker, A. B., Bates, S. R. G., Llewellyn-Jones, T. M., Valori, L. P. B., Dicker, M. P. M., and Trask, R. S. (2019). 4D Printing with Robust Thermoplastic Polyurethane Hydrogel-Elastomer Trilayers. Mater. Des. 163, 107544. doi:10.1016/j.matdes. 2018.107544

Banerjee, H., Suhail, M., and Ren, H. (2018). Hydrogel Actuators and Sensors for Biomedical Soft Robots: Brief Overview with Impending Challenges. Biomimetics 3, 15-41. doi:10.3390/biomimetics3030015

Billiet, T., Vandenhaute, M., Schelfhout, J., Van Vlierberghe, S., and Dubruel, P. (2012). A Review of Trends and Limitations in Hydrogel-Rapid Prototyping for Tissue Engineering. Biomaterials 33, 6020-6041. doi:10.1016/j.biomaterials. 2012.04.050 resolutions of specific printing techniques. To address these challenges and broaden the areas of application for $3 \mathrm{D}$ printing hydrogel actuators, future research is needed to integrate multiple $3 \mathrm{D}$ fabrication techniques and utilize the combined strengths of individual methods in a single platform. For example, a hydrogel robot may require features with different spatial resolutions at different locations on the robot body. To accomplish this, an extrusion-based method could print bulk material for features with low resolution and laser-based methods could make features with high resolution. Such integrated platforms could also facilitate multi-material integration for additional functionality. For example, embedded printing methods could introduce traces of conductive and stimuli-responsive material into hydrogel systems to create actuators with embedded sensors. Addressing these challenges through innovative fabrication techniques will further improve the performance of hydrogel-based actuators for applications in soft robotics.

\section{AUTHOR CONTRIBUTIONS}

WS, LY, AF, and VWW conceptualized and outlined this review. WS drafted section 1, 2, 3.3, 3.5, 5, VWW drafted section 3.2, $\mathrm{KD}$, drafted section 3.1, 3.4, S.S. drafted section 4. WS, KD, SS, LY, AF, and VWW edited the manuscript.

\section{FUNDING}

This work was supported by the National Science Foundation (NSF) Research Fellowship Program under Grant No. DGE1745016 and NSF Next Generation Networks for Neuroscience (NeuroNex) Program under Grant No. DBI2015317. Any opinions, findings, and conclusions or recommendations expressed in this material are those of the author(s) and do not necessarily reflect the views of the National Science Foundation.

Bozuyuk, U., Yasa, O., Yasa, I. C., Ceylan, H., Kizilel, S., and Sitti, M. (2018). LightTriggered Drug Release from 3D-Printed Magnetic Chitosan Microswimmers. ACS Nano 12, 9617-9625. doi:10.1021/acsnano.8b05997

Breuer, L., Pilas, J., Guthmann, E., Schöning, M. J., Thoelen, R., and Wagner, T. (2019). Towards Light-Addressable Flow Control: Responsive Hydrogels with Incorporated Graphene Oxide as Laser-Driven Actuator Structures within Microfluidic Channels. Sensors Actuators B: Chem. 288, 579-585. doi:10.1016/j.snb.2019.02.086

Cabanach, P., Pena-Francesch, A., Sheehan, D., Bozuyuk, U., Yasa, O., Borros, S., et al. (2020). Zwitterionic 3D-Printed Non-Immunogenic Stealth Microrobots. Adv. Mater. 32, 2003013. doi:10.1002/adma.202003013

Ceylan, H., Yasa, I. C., Yasa, O., Tabak, A. F., Giltinan, J., and Sitti, M. (2019). 3DPrinted Biodegradable Microswimmer for Theranostic Cargo Delivery and Release. ACS Nano 13, 3353-3362. doi:10.1021/acsnano.8b09233

Champeau, M., Heinze, D. A., Viana, T. N., de Souza, E. R., Chinellato, A. C., and Titotto, S. (2020). 4D Printing of Hydrogels: A Review. Adv. Funct. Mater. 30, 1910606-1910622. doi:10.1002/adfm.201910606

Chan, V., Jeong, J. H., Bajaj, P., Collens, M., Saif, T., Kong, H., et al. (2012). Multimaterial Bio-Fabrication of Hydrogel Cantilevers and Actuators with Stereolithography. Lab. Chip 12, 88-98. doi:10.1039/c1lc20688e 
Chen, T., Bakhshi, H., Liu, L., Ji, J., and Agarwal, S. (2018). Combining 3D Printing with Electrospinning for Rapid Response and Enhanced Designability of Hydrogel Actuators. Adv. Funct. Mater. 28, 1800514-1800519. doi:10.1002/ adfm.201800514

Cheng, Y., Chan, K. H., Wang, X.-Q., Ding, T., Li, T., Lu, X., et al. (2019). DirectInk-Write 3D Printing of Hydrogels into Biomimetic Soft Robots. ACS Nano 13, 13176-13184. doi:10.1021/acsnano.9b06144

Chin, S. Y., Poh, Y. C., Kohler, A.-C., Compton, J. T., Hsu, L. L., Lau, K. M., et al. (2017). Additive Manufacturing of Hydrogel-Based Materials for NextGeneration Implantable Medical Devices. Sci. Robotics 2. doi:10.1126/ scirobotics.aah6451

Christensen, K., Xu, C., Chai, W., Zhang, Z., Fu, J., and Huang, Y. (2015). Freeform Inkjet Printing of Cellular Structures with Bifurcations. Biotechnol. Bioeng. 112, 1047-1055. doi:10.1002/bit.25501

Cvetkovic, C., Raman, R., Chan, V., Williams, B. J., Tolish, M., Bajaj, P., et al. (2014). Three-dimensionally Printed Biological Machines Powered by Skeletal Muscle. Proc. Natl. Acad. Sci. 111, 10125-10130. doi:10.1073/pnas.1401577111

de Marco, C., Alcântara, C. C. J., Kim, S., Briatico, F., Kadioglu, A., de Bernardis, G., et al. (2019). Indirect 3D and 4D Printing of Soft Robotic Microstructures. $A d v$. Mater. Technol. 4, 1900332-1900337. doi:10.1002/admt.201900332

Dong, M., Wang, X., Chen, X. Z., Mushtaq, F., Deng, S., Zhu, C., et al. (2020). 3DPrinted Soft Magnetoelectric Microswimmers for Delivery and Differentiation of Neuron-Like Cells. Adv. Funct. Mater. 30, 1910323-1910327. doi:10.1002/ adfm.201910323

Erol, O., Pantula, A., Liu, W., and Gracias, D. H. (2019). Transformer Hydrogels: A Review. Adv. Mater. Technol. 4, 1900043. doi:10.1002/admt.201900043

Fornell, A., Johannesson, C., Searle, S. S., Happstadius, A., Nilsson, J., and Tenje, M. (2019). An Acoustofluidic Platform for Non-contact Trapping of Cell-Laden Hydrogel Droplets Compatible with Optical Microscopy. Biomicrofluidics 13, 044101. doi:10.1063/1.5108583

Gladman, A. S., Matsumoto, E. A., Nuzzo, R. G., Mahadevan, L., and Lewis, J. A. (2016). Biomimetic 4D Printing. Nat. Mater. 15, 413-418. doi:10.1038/ nmat4544

Guillemot, F., Guillotin, B., Fontaine, A., Ali, M., Catros, S., Kériquel, V., et al. (2011). Laser-assisted Bioprinting to Deal with Tissue Complexity in Regenerative Medicine. MRS Bull. 36, 1015-1019. doi:10.1557/mrs. 2011.272

Guo, F., Mao, Z., Chen, Y., Xie, Z., Lata, J. P., Li, P., et al. (2016). Three-dimensional Manipulation of Single Cells Using Surface Acoustic Waves. Proc. Natl. Acad. Sci. USA 113, 1522-1527. doi:10.1073/pnas.1524813113

Hamedi, M. M., Campbell, V. E., Rothemund, P., Güder, F., Christodouleas, D. C., Bloch, J.-F., et al. (2016). Electrically Activated Paper Actuators. Adv. Funct. Mater. 26, 2446-2453. doi:10.1002/adfm.201505123

Han, D., Farino, C., Yang, C., Scott, T., Browe, D., Choi, W., et al. (2018). Soft Robotic Manipulation and Locomotion with a 3D Printed Electroactive Hydrogel. ACS Appl. Mater. Inter. 10, 17512-17518. doi:10.1021/acsami. $8 \mathrm{~b} 04250$

Hinton, T. J., Jallerat, Q., Palchesko, R. N., Park, J. H., Grodzicki, M. S., Shue, H.-J., et al. (2015). Three-dimensional Printing of Complex Biological Structures by Freeform Reversible Embedding of Suspended Hydrogels. Sci. Adv. 1, e1500758-10. doi:10.1126/sciadv.1500758

Hippler, M., Blasco, E., Qu, J., Tanaka, M., Barner-Kowollik, C., Wegener, M., et al. (2019). Controlling the Shape of 3D Microstructures by Temperature and Light. Nat. Commun. 10, 1-8. doi:10.1038/s41467-018-08175-w

Hua, L., Xie, M., Jian, Y., Wu, B., Chen, C., and Zhao, C. (2019). Multipleresponsive and Amphibious Hydrogel Actuator Based on Asymmetric Ucsttype Volume Phase Transition. ACS Appl. Mater. Inter. 11, 43641-43648. doi:10.1021/acsami.9b17159

Huang, T.-Y., Huang, H.-W., Jin, D. D., Chen, Q. Y., Huang, J. Y., Zhang, L., et al. (2020). Four-dimensional Micro-building Blocks. Sci. Adv. 6, eaav8219-10. doi:10.1126/sciadv.aav8219

Jakab, K., Norotte, C., Marga, F., Murphy, K., Vunjak-Novakovic, G., and Forgacs, G. (2010). Tissue Engineering by Self-Assembly and Bio-Printing of Living Cells. Biofabrication 2, 022001. doi:10.1088/1758-5082/2/2/ 022001

Jin, D., Chen, Q., Huang, T.-Y., Huang, J., Zhang, L., and Duan, H. (2020). Fourdimensional Direct Laser Writing of Reconfigurable Compound Micromachines. Mater. Today 32, 19-25. doi:10.1016/j.mattod.2019.06.002
Kaynak, M., Dirix, P., and Sakar, M. S. (2020). Addressable Acoustic Actuation of 3D Printed Soft Robotic Microsystems. Adv. Sci. 7, 2001120-2001129. doi:10. 1002/advs.202001120

Koepele, C. A., Guix, M., Bi, C., Adam, G., and Cappelleri, D. J. (2020). 3D-Printed Microrobots with Integrated Structural Color for Identification and Tracking. Adv. Intell. Syst. 2, 1900147. doi:10.1002/aisy.201900147

Kramb, R. C., Buskohl, P. R., Slone, C., Smith, M. L., and Vaia, R. A. (2014). Autonomic Composite Hydrogels by Reactive Printing: Materials and Oscillatory Response. Soft Matter 10, 1329-1336. doi:10.1039/c3sm51650d

Kyle, S., Jessop, Z. M., Al-Sabah, A., and Whitaker, I. S. (2017). 'Printability' of Candidate Biomaterials for Extrusion Based 3D Printing: State-Of-The-Art. Adv. Healthc. Mater. 6, 1700264. doi:10.1002/adhm.201700264

Le, X., Lu, W., Zhang, J., and Chen, T. (2019). Recent Progress in Biomimetic Anisotropic Hydrogel Actuators. Adv. Sci. 6, 1801584. doi:10.1002/advs. 201801584

Lee, A., Hudson, A. R., Shiwarski, D. J., Tashman, J. W., Hinton, T. J., Yerneni, S., et al. (2019). 3D Bioprinting of Collagen to Rebuild Components of the Human Heart. Science 365, 482-487. doi:10.1126/science.aav9051

Lee, Y., Song, W. J., and Sun, J.-Y. (2020). Hydrogel Soft Robotics. Mater. Today Phys. 15, 100258. doi:10.1016/j.mtphys.2020.100258

Li, J., and Mooney, D. J. (2016). Designing Hydrogels for Controlled Drug Delivery. Nat. Rev. Mater. 1. doi:10.1038/natrevmats.2016.71

Li, J., Wu, C., Chu, P. K., and Gelinsky, M. (2020). 3D Printing of Hydrogels: Rational Design Strategies and Emerging Biomedical Applications. Mater. Sci. Eng. R: Rep. 140, 100543. doi:10.1016/j.mser.2020.100543

Lin, Q., Tang, M., and Ke, C. (2020). Thermo-responsive 3d-Printed Polyrotaxane Monolith. Polym. Chem. 11, 304-308. doi:10.1039/c9py01510h

Lv, C., Sun, X.-C., Xia, H., Yu, Y.-H., Wang, G., Cao, X.-W., et al. (2018). Humidityresponsive Actuation of Programmable Hydrogel Microstructures Based on 3d Printing. Sensors Actuators B: Chem. 259, 736-744. doi:10.1016/j.snb.2017. 12.053

Malda, J., Visser, J., Melchels, F. P., Jüngst, T., Hennink, W. E., Dhert, W. J. A., et al. (2013). 25th Anniversary Article: Engineering Hydrogels for Biofabrication. Adv. Mater. 25, 5011-5028. doi:10.1002/adma.201302042

Mantha, S., Pillai, S., Khayambashi, P., Upadhyay, A., Zhang, Y., Tao, O., et al. (2019). Smart Hydrogels in Tissue Engineering and Regenerative Medicine. Materials 12, 3323. doi:10.3390/ma12203323

Marzban, B., and Yuan, H. (2016). The Effect of Viscous Force on the Prediction of Muscle Contractility in Biohybrid Cantilever-Based Experiments. Extreme Mech. Lett. 9, 342-346. doi:10.1016/j.eml.2016.09.011

Matouš, J., Kollarčik, A., Gurtner, M., Michálek, T., and Hurák, Z. (2019). Optimization-based Feedback Manipulation through an Array of Ultrasonic Transducers. IFAC-PapersOnLine 52, 483-488. doi:10.1016/j.ifacol.2019.11.722

Mestre, R., Patiño, T., Guix, M., Barceló, X., and Sanchez, S. (2019). Design, Optimization and Characterization of Bio-Hybrid Actuators Based on 3dBioprinted Skeletal Muscle Tissue. Conference on Biomimetic and Biohybrid Systems. Springer, 205-215. doi:10.1007/978-3-030-24741-6_18

Mirdamadi, E., Tashman, J. W., Shiwarski, D. J., Palchesko, R. N., and Feinberg, A. W. (2020). FRESH 3D Bioprinting a Full-Size Model of the Human Heart. ACS Biomater. Sci. Eng. 6, 6453-6459. doi:10.1021/acsbiomaterials.0c01133

Mishra, A. K., Wallin, T. J., Pan, W., Xu, P., Wang, K., Giannelis, E. P., et al. (2020). Autonomic Perspiration in 3D-Printed Hydrogel Actuators. Sci. Robot. 5, eaaz3918-10. doi:10.1126/scirobotics.aaz3918

Momeni, F., S.M. Mehdi Hassani. N, S., Liu, X., and Ni, J. (2017). A Review of 4D Printing. Mater. Des. 122, 42-79. doi:10.1016/j.matdes.2017.02.068

Morimoto, Y., and Takeuchi, S. (2020). Biohybrid Robot Powered by Muscle Tissues. Mechanically Responsive Mater. Soft Robotics 4, 395-416. doi:10.1002/ 9783527822201.ch16

Nadgorny, M., Xiao, Z., Chen, C., and Connal, L. A. (2016). Three-Dimensional Printing of $\mathrm{pH}$-Responsive and Functional Polymers on an Affordable Desktop Printer. ACS Appl. Mater. Inter. 8, 28946-28954. doi:10.1021/acsami.6b07388

Naficy, S., Gately, R., Gorkin, R., Xin, H., and Spinks, G. M. (2017). 4D Printing of Reversible Shape Morphing Hydrogel Structures. Macromol. Mater. Eng. 302, 1600212-1600219. doi:10.1002/mame.201600212

Nishiguchi, A., Zhang, H., Schweizerhof, S., Schulte, M. F., Mourran, A., and Möller, M. (2020). 4D Printing of a Light-Driven Soft Actuator with Programmed Printing Density. ACS Appl. Mater. Inter. 12, 12176-12185. doi:10.1021/acsami.0c02781 
Odent, J., Vanderstappen, S., Toncheva, A., Pichon, E., Wallin, T. J., Wang, K., et al. (2019). Hierarchical Chemomechanical Encoding of Multi-Responsive Hydrogel Actuators via 3D Printing. J. Mater. Chem. A. 7, 15395-15403. doi:10.1039/c9ta03547h

Orbay, S., Ozcelik, A., Bachman, H., and Huang, T. J. (2018). Acoustic Actuation of in situ Fabricated Artificial Cilia.

Ovsianikov, A., Gruene, M., Pflaum, M., Koch, L., Maiorana, F., Wilhelmi, M., et al. (2010). Laser Printing of Cells into 3D Scaffolds. Biofabrication 2, 014104. doi:10.1088/1758-5082/2/1/014104

Ozcelik, A., Rufo, J., Guo, F., Gu, Y., Li, P., Lata, J., et al. (2018). Acoustic Tweezers for the Life Sciences. Nat. Methods 15, 1021-1028. doi:10.1038/s41592-0180222-9

Pagan-Diaz, G. J., Zhang, X., Grant, L., Kim, Y., Aydin, O., Cvetkovic, C., et al. (2018). Simulation and Fabrication of Stronger, Larger, and Faster Walking Biohybrid Machines. Adv. Funct. Mater. 28, 1801145. doi:10.1002/adfm. 201801145

Pan, D., Wu, D., Li, P. J., Ji, S. Y., Nie, X., Fan, S. Y., et al. (2021). Transparent LightDriven Hydrogel Actuator Based on Photothermal Marangoni Effect and Buoyancy Flow for Three-Dimensional Motion. Adv. Funct. Mater. 31, 2009386-2009389. doi:10.1002/adfm.202009386

Park, J., Jin, C., Lee, S., Kim, J. Y., and Choi, H. (2019). Magnetically Actuated Degradable Microrobots for Actively Controlled Drug Release and Hyperthermia Therapy. Adv. Healthc. Mater. 8, 1900213. doi:10.1002/adhm. 201900213

Peng, X., Liu, T., Zhang, Q., Shang, C., Bai, Q.-W., and Wang, H. (2017). Surface Patterning of Hydrogels for Programmable and Complex Shape Deformations by Ion Inkjet Printing. Adv. Funct. Mater. 27, 1701962-1701968. doi:10.1002/ adfm.201701962

Peng, X., and Wang, H. (2018). Shape Changing Hydrogels and Their Applications as Soft Actuators. J. Polym. Sci. Part. B: Polym. Phys. 56, 1314-1324. doi:10. 1002/polb. 24724

Podstawczyk, D., Nizioł, M., Szymczyk, P., Wiśniewski, P., and Guiseppi-Elie, A. (2020). 3D Printed Stimuli-Responsive Magnetic Nanoparticle Embedded Alginate-Methylcellulose Hydrogel Actuators. Addit. Manuf. 34, 101275. doi:10.1016/j.addma.2020.101275

Raman, R., Cvetkovic, C., Uzel, S. G. M., Platt, R. J., Sengupta, P., Kamm, R. D., et al. (2016). Optogenetic Skeletal Muscle-Powered Adaptive Biological Machines. Proc. Natl. Acad. Sci. USA 113, 3497-3502. doi:10.1073/pnas.1516139113

Raviv, D., Zhao, W., McKnelly, C., Papadopoulou, A., Kadambi, A., Shi, B., et al. (2014). Active Printed Materials for Complex Self-Evolving Deformations. Sci. Rep. 4, 1-9. doi:10.1038/srep07422

Rehor, I., Maslen, C., Moerman, P. G., van Ravensteijn, B. G. P., van Alst, R., Groenewold, J., et al. (2021). Photoresponsive Hydrogel Microcrawlers Exploit Friction Hysteresis to Crawl by Reciprocal Actuation. Soft Robotics 8, 10-18. doi:10.1089/soro.2019.0169

Ren, L., Nama, N., McNeill, J. M., Soto, F., Yan, Z., Liu, W., et al. (2019). 3D Steerable, Acoustically Powered Microswimmers for Single-Particle Manipulation. Sci. Adv. 5, eaax3084. doi:10.1126/sciadv.aax3084

Ricotti, L., and Fujie, T. (2017). Thin Polymeric Films for Building Biohybrid Microrobots. Bioinspir. Biomim. 12, 021001. doi:10.1088/1748-3190/aa5e5f

Ricotti, L., Trimmer, B., Feinberg, A. W., Raman, R., Parker, K. K., Bashir, R., et al. (2017). Biohybrid Actuators for Robotics: A Review of Devices Actuated by Living Cells. Sci. Robot. 2, eaaq0495-18. doi:10.1126/scirobotics.aaq0495

Rivera, M. L., Forman, J., Hudson, S. E., and Yao, L. (2020). "Hydrogel-Textile Composites," in Proceedings of the $2020 \mathrm{CHI}$ Conference on Human Factors in Computing Systems, 1-9. doi:10.1145/3334480.3382788

Shang, J., Le, X., Zhang, J., Chen, T., and Theato, P. (2019). Trends in Polymeric Shape Memory Hydrogels and Hydrogel Actuators. Polym. Chem. 10, 1036-1055. doi:10.1039/c8py01286e

Shi, Q., Liu, H., Tang, D., Li, Y., Li, X., and Xu, F. (2019). Bioactuators Based on Stimulus-Responsive Hydrogels and Their Emerging Biomedical Applications. NPG Asia Mater. 11. doi:10.1038/s41427-019-0165-3

Soman, P., Chung, P. H., Zhang, A. P., and Chen, S. (2013). Digital Microfabrication of User-Defined 3D Microstructures in Cell-Laden Hydrogels. Biotechnol. Bioeng. 110, 3038-3047. doi:10.1002/bit.24957

Son, H., Byun, E., Yoon, Y. J., Nam, J., Song, S. H., and Yoon, C. (2020). Untethered Actuation of Hybrid Hydrogel Gripper via Ultrasound. ACS Macro Lett. 9, 1766-1772. doi:10.1021/acsmacrolett.0c00702
Spiegel, C. A., Hippler, M., Münchinger, A., Bastmeyer, M., Barner-Kowollik, C., Wegener, M., et al. (2020). 4D Printing at the Microscale. Adv. Funct. Mater. 30, 1907615. doi:10.1002/adfm.201907615

Sun, L., Yu, Y., Chen, Z., Bian, F., Ye, F., Sun, L., et al. (2020). Biohybrid Robotics with Living Cell Actuation. Chem. Soc. Rev. Chemical Society Reviews. 49, 4043-4069. doi:10.1039/D0CS00120A

Tao, X., Nguyen, T. D., Jin, H., Tao, R., Luo, J., Yang, X., et al. (2019). 3D Patterning/manipulating Microparticles and Yeast Cells Using $\mathrm{ZnO} / \mathrm{Si}$ Thin Film Surface Acoustic Waves. Sensors Actuators B: Chem. 299, 126991. doi:10. 1016/j.snb.2019.126991

Tognato, R., Armiento, A. R., Bonfrate, V., Levato, R., Malda, J., Alini, M., et al. (2019). A Stimuli-Responsive Nanocomposite for 3D Anisotropic CellGuidance and Magnetic Soft Robotics. Adv. Funct. Mater. 29, 1804647. doi:10.1002/adfm.201804647

Tyagi, M., Spinks, G. M., and Jager, E. W. H. (2020). Fully 3D Printed Soft Microactuators for Soft Microrobotics. Smart Mater. Struct. 29, 085032. doi:10. 1088/1361-665X/ab9f48

Varaprasad, K., Jayaramudu, T., Kanikireddy, V., Toro, C., and Sadiku, E. R. (2020). Alginate-based Composite Materials for Wound Dressing Application: A Mini Review. Carbohydr. Polym. 236, 116025. doi:10.1016/j.carbpol.2020. 116025

Wallin, T. J., Pikul, J., and Shepherd, R. F. (2018). 3D Printing of Soft Robotic Systems. Nat. Rev. Mater. 3, 84-100. doi:10.1038/s41578-018-0002-2

Wang, W., Yao, L., Cheng, C.-Y., Zhang, T., Atsumi, H., Wang, L., et al. (2017). Harnessing the Hygroscopic and Biofluorescent Behaviors of Genetically Tractable Microbial Cells to Design Biohybrid Wearables. Sci. Adv. 3, e1601984. doi:10.1126/sciadv.1601984

Wang, X., Qin, X.-H., Hu, C., Terzopoulou, A., Chen, X.-Z., Huang, T.-Y., et al. (2018). 3D Printed Enzymatically Biodegradable Soft Helical Microswimmers. Adv. Funct. Mater. 28, 1804107-1804108. doi:10.1002/adfm.201804107

Watanabe, T., Akiyama, M., Totani, K., Kuebler, S. M., Stellacci, F., Wenseleers, W., et al. (2002). Photoresponsive Hydrogel Microstructure Fabricated by TwoPhoton Initiated Polymerization. Adv. Funct. Mater. 12, 611-614. doi:10.1002/ 1616-3028(20020916)12:9<611::AID-ADFM611>3.0.CO;2-3

Webster-Wood, V. A., Akkus, O., Gurkan, U. A., Chiel, H. J., and Quinn, R. D. (2017). Organismal Engineering: Toward a Robotic Taxonomic Key for Devices Using Organic Materials. Sci. Robot. 2, eaap9281. doi:10.1126/scirobotics.aap9281

Won, P., Ko, S. H., Majidi, C., W. Feinberg, A., and A. Webster-Wood, V. (2020). "Biohybrid Actuators for Soft Robotics: Challenges in Scaling up. Actuators 9, 96. doi:10.3390/act9040096

Xing, J.-F., Zheng, M.-L., and Duan, X.-M. (2015). Two-photon Polymerization Microfabrication of Hydrogels: an Advanced 3D Printing Technology for Tissue Engineering and Drug Delivery. Chem. Soc. Rev. 44, 5031-5039. doi: $10.1039 / \mathrm{c} 5 \mathrm{cs} 00278 \mathrm{~h}$

Xiong, Z., Zheng, M.-L., Dong, X.-Z., Chen, W.-Q., Jin, F., Zhao, Z.-S., et al. (2011). Asymmetric Microstructure of Hydrogel: Two-Photon Microfabrication and Stimuli-Responsive Behavior. Soft Matter 7, 10353-10359. doi:10.1039/ clsm06137b

Yao, L., Ou, J., Cheng, C.-Y., Steiner, H., Wang, W., Wang, G., et al. (2015). "bioLogic: Natto Cells as Nanoactuators for Shape Changing Interfaces Lining," in Proceedings of the 2015 CHI Conference on Human Factors in Computing Systems - CHI '15, 567-572. doi:10.1017/ CBO9781139855952.100

Yuk, H., Lin, S., Ma, C., Takaffoli, M., Fang, N. X., and Zhao, X. (2017). Hydraulic Hydrogel Actuators and Robots Optically and Sonically Camouflaged in Water. Nat. Commun. 8. doi:10.1038/ncomms14230

Zhang, Y., Ellison, S. T., Duraivel, S., Morley, C. D., Taylor, C. R., and Angelini, T. E. (2021). 3D Printed Collagen Structures at Low Concentrations Supported by Jammed Microgels. Bioprinting 21, e00121. doi:10.1016/j.bprint.2020.e00121

Zhang, Y. S., and Khademhosseini, A. (2017). Advances in Engineering Hydrogels. Science 356. doi:10.1126/science.aaf3627

Zhao, Q., Liang, Y., Ren, L., Yu, Z., Zhang, Z., and Ren, L. (2018). Bionic Intelligent Hydrogel Actuators with Multimodal Deformation and Locomotion. Nano Energy 51, 621-631. doi:10.1016/j.nanoen.2018.07.025

Zheng, C., Jin, F., Zhao, Y., Zheng, M., Liu, J., Dong, X., et al. (2020). Light-driven Micron-Scale 3d Hydrogel Actuator Produced by Two-Photon Polymerization Microfabrication. Sensors Actuators B: Chem. 304, 127345. doi:10.1016/j.snb. 2019.127345 
Zheng, S. Y., Shen, Y., Zhu, F., Yin, J., Qian, J., Fu, J., et al. (2018). Programmed Deformations of 3D-Printed Tough Physical Hydrogels with High Response Speed and Large Output Force. Adv. Funct. Mater. 28, 1803366-1803368. doi:10.1002/adfm.201803366

Zhu, W., Li, J., Leong, Y. J., Rozen, I., Qu, X., Dong, R., et al. (2015). 3D-Printed Artificial Microfish. Adv. Mater. 27, 4411-4417. doi:10.1002/adma.201501372

Zolfagharian, A., Kouzani, A. Z., Khoo, S. Y., Gibson, I., and Kaynak, A. (2017a). 3D Printed Hydrogel Soft Actuators. Proceedings/TENCON 2016, 2272-2277. doi:10.1109/TENCON.2016.7848433

Zolfagharian, A., Kouzani, A. Z., Khoo, S. Y., Nasri-Nasrabadi, B., and Kaynak, A. (2017b). Development and Analysis of a 3d Printed Hydrogel Soft Actuator. Sensors Actuators A: Phys. 265, 94-101. doi:10.1016/j.sna.2017.08.038
Conflict of Interest: The authors declare that the research was conducted in the absence of any commercial or financial relationships that could be construed as a potential conflict of interest.

Copyright (c) 2021 Sun, Schaffer, Dai, Yao, Feinberg and Webster-Wood. This is an open-access article distributed under the terms of the Creative Commons Attribution License (CC BY). The use, distribution or reproduction in other forums is permitted, provided the original author(s) and the copyright owner(s) are credited and that the original publication in this journal is cited, in accordance with accepted academic practice. No use, distribution or reproduction is permitted which does not comply with these terms. 